\title{
ÉTUDE DES VARIATIONS DES COMMUNAUTÉS VÉGÉTALES AQUATIQUES SOUS L'EFFET DE PERTURBATIONS ANTHROPIQUES EN RIVIÈRE RÉGULÉE.
}

\section{BERNEZ, H. DANIEL* et J. HAURY}

ENSAR, Laboratoire d'Ecologie et Sciences Phytosanitaires, INRA, Laboratoire d'Ecologie Aquatique, 65 rue de St-Brieuc, 35042 Rennes cedex.

* adresse actuelle / present adress : SCE, Dpt Eau, Atlanpole, site de la Chantrerie, route de Gachet, BP 10703, 44307 Nantes cedex 3.

\section{RÉSUMÉ}

Les macrophytes de la Rance sont étudiés selon le protocole d'échantillonnage «Milieu Et Végétation aquatique fixée (MEV)» des Agences de l'Eau. Cette rivière armoricaine subit des pollutions industrielles, agricoles et perturbations hydrauliques marquées.

Les variations spatio-temporelles des communautés végétales échantillonnées sont étudiées au travers de la richesse floristique, d'indices de diversité et d'équitabilité, d'indices biologiques macrophytiques et des recouvrements des groupes et types biologiques. pertinent.

Le protocole $\mathrm{MEV}$, en premier essai dans des secteurs régulés, s'avère être

La structure de la végétation aquatique rend compte des perturbations liées au mode de gestion du barrage EDF de Rophémel. Les pollutions à l'amont, liées à des problèmes de qualité chimique de l'eau, sont également repérées par les modifications des communautés.

Les indices biologiques macrophytiques rendent compte des modifications saisonnières liées à l'étiage estival. Dans le contexte très anthropisé de la Rance, leur possibilité d'usage reste limitée.

Des croissances proliférantes des végétaux sont observées à l'étiage. Les secteurs repérés comme perturbés sont les plus affectés. Ces déséquilibres de structure des communautés végétales et leurs variations saisonnières contribuent à la compréhension du fonctionnement en rivière.

Les perspectives d'amélioration de ces indices passent par un approfondissement analytique et conceptuel. Une expérimentation in situ, en se servant des particularités des milieux régulés, est envisagée pour le perfectionnement du suivi hydrobiologique des rivières.

Mots-clés : macrophytes, écohydraulique, barrage hydroélectrique, perturbation, échantillonnage, MEV, indice biologique macrophytique, indices de structure. 


\section{EFFECTS OF PERTURBATIONS ON THE AQUATIC VEGETATION OF REGULATED RIVER.}

\section{ABSTRACT}

Macrophytes of the River Rance were studied according to the sampling methods of the French Water Agencies ("protocole MEV") : survey of $50 \mathrm{~m}$ length stations along the river gradient; the sectorisation of the river was done considering abiotic factors such as drainage order, slope, geology and pollution points.

Spatio-temporal variations of the floristic communities were described through species richness, diversity and evenness indexes, macrophyte index and cover of the types and the biological groups.

For its first application in regulated parts, MEV protocol gave quite a good result.

Floristic structure changed according to the management of the hydroelectric dam of Rophémel. Upstream, chemical industrial and agricultural pollution modified the vegetation too. The more obvious modification occurred for the hydraulic perturbation.

This response was obtained with the French macrophyte index too (Indice Biologique Macrophytique : IBM) : The low waters of September decreased the notes of the index. Several propositions are discussed to improve the index according to the English methodology developed trough the Mean Trophic Rank.

Great macrophytes cover was observed at the low water level of the end of the summer. Perturbed parts were more affected. The balance between community structures gave a functional indication of the River Rance.

The comparison of seasonal variations occurs to be a way of study of the biological response to perturbation. According to the great response of the vegetation downstream from the dam, perspectives are proposed in using regulated parts of the river as an in situ experimental tool to increase the understanding of ecological mechanisms.

Key-words : river macrophytes, ecohydraulics, dam, perturbation, sampling, MEV, plant index, structure index.

\section{INTRODUCTION}

Les données biologiques sont de plus en plus utilisées pour la surveillance de l'environnement. La raison du développement de ces études biologiques est qu'elles traduisent les perturbations sur les écosystèmes et contiennent une information synthétique (COUILLARD et LEFEBVRE, 1985 ; COURTEMANCH et DAVIES, 1987). La végétation est un compartiment privilégié de par le rôle d'habitat, le rôle trophique qu'elle joue et les facilités d'observation qu'elle permet (HASLAM, 1978). Des besoins d'évaluation de l'état de santé des rivières ont amené à développer des outils de bio-évaluation (CAIRNS et al., 1993). En France, des études des perturbations ont porté sur des secteurs soumis à des variations hydrauliques saisonnières (HENRY et AMOROS, 1996), à des régulations hydrauliques (comme les avals de barrages). Dans ce dernier contexte, les plantes aquatiques ont été étudiées par rapport à des problèmes de proliférations d'herbiers sur la Dordogne et le Vair en relation avec la qualité de l'eau (GOSSE, 1989 ; PETITJEAN, 1981), ou pour des études de dynamique des populations algales sur la Durance (FAYOLLE, 1998). Dans le Massif armoricain, des études sur les plantes aquatiques des rivières sont réalisées depuis 1979 par HAURY (1985). Les travaux en secteurs régulés y sont plus récents (BERNEZ, 1999 ; HAURY et al., 1996a). 
L'objectif de l'étude présentée est double. II s'agit premièrement de suivre sur une rivière de Bretagne l'impact des perturbations sur la végétation. Deuxièmement, nous envisageons d'utiliser et comparer des méthodologies développées pour les Agences de l'Eau, tant au niveau de l'échantillonnage que des moyens d'interprétation : les variations spatio-temporelles des communautés végétales sont analysées à l'aide d'indices de diversité des peuplements, d'Indices Biologiques Macrophytiques, et enfin les caractéristiques stationnelles du recouvrement végétal : c'est un premier essai en rivière très perturbée, notamment par des régulations, du protocole d'échantillonnage « Milieu Et Végétation aquatique fixée " (HAURY et al., 1998) et des indices biologiques macrophytiques (HAURY et al., 1996b).

Lintérêt d'une telle approche est de permettre d'élargir le potentiel de gestion écologique des rivières du point de vue de leur végétation.

\section{MÉTHODOLOGIE}

\section{Sites}

\section{La Rance}

La Rance, fleuve du nord-est de la Bretagne (Figure 1) est classée en première catégorie piscicole sur le tiers amont de la zone d'étude, puis en deuxième catégorie. La partie étudiée du cours a une longueur de $67 \mathrm{~km}$, des sources jusqu'au canal d'llle-et-Rance. Dans le tiers aval de ces $67 \mathrm{~km}$, on trouve une modification hydraulique majeure, le barrage de Rophémel (4,9 millions de $\mathrm{m}^{3}$, débit moyen annuel : $2,74 \mathrm{~m}^{3} / \mathrm{s}$, débit réservé : $0,1 \mathrm{~m}^{3} / \mathrm{s}$, débit maximum turbinable : $27 \mathrm{~m}^{3} / \mathrm{s}$, crue maximale observée : $120 \mathrm{~m}^{3} / \mathrm{s}$ ) : construit en 1936, il est classé comme grand barrage. Son usage actuel est double, il sert depuis sa construction à la production d'hydroélectricité et maintenant, son usage prioritaire est l'alimentation en eau potable pour la ville de Rennes. Ceci a des incidences sur la fréquence des éclusées en été : seulement deux dates de turbinage en août 1995. Ceci donne une physionomie particulière à la Rance à l'aval du site hydroélectrique, où elle possède une partie chenalisée surdimensionnée pour recevoir les débits turbinés. En plus de ces perturbations physiques, la Rance subit l'effet d'effluents chimiques dès ses sources à Collinée (22). Dans cette ville sont implantés les plus gros abattoirs industriels de Bretagne. Lorsqu'elle en traverse les lagunes, la rivière est à un ordre 1 de drainage de STRAHLER (1964, in BEAUMONT, 1975). Tout le bassin, dès l'amont, subit aussi une agriculture intensive.

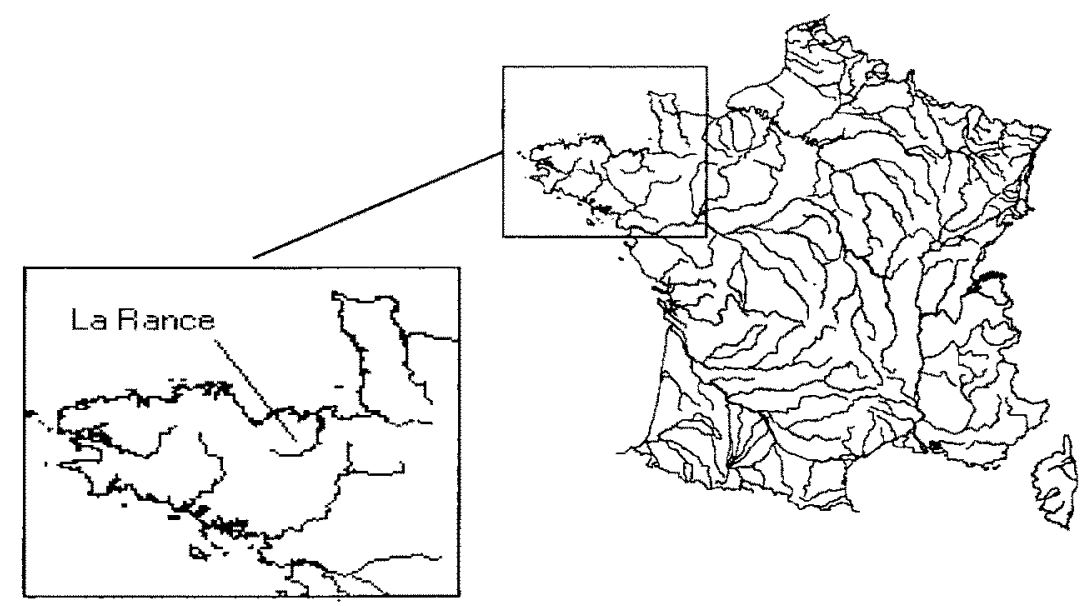

Figure 1

Localisation de la Rance.

\section{Figure 1}




\section{Echantillonnage}

La méthode d'acquisition des données suit le protocole "Milieu Et Végétation aquatique fixée " (MEV) (HAURY et al., 1998). Un découpage de la rivière en tronçons, dit " découpage abiotique ", est réalisé selon le protocole hiérarchisé préconisé (LEGLIZE et al., 1990), à chaque modification de l'ordre de drainage, de la pente, de la géologie ou bien lors de la présence d'un point de pollution. Dans le tiers aval de ces tronçons des stations sont choisies. Les recouvrements floristiques (plantes vasculaires, bryophytes et macro-algues déterminées à l'espèce ou au genre) de 44 stations (début d'été : juin/juillet) et de 30 stations communes à cet échantillonnage de juillet sont réalisés en fin d'été (septembre). En juillet des stations ont été dédoublées pour tester l'échantillonnage, ceci justifie les 14 stations supplémentaires. Les stations font au moins $50 \mathrm{~m}$ de longueur et sont réparties sur tout le linéaire de la Rance. Ces deux campagnes optimisent la liste d'espèces en s'affranchissant des comportements saisonniers (HAURY et al., 1998). Pour les comparaisons saisonnières, on mettra en parallèle les relevés des 30 stations communes aux deux campagnes.

Le protocole "MEV" inclut aussi une phase d'acquisition de données physico-chimiques qui permet d'envisager les relations plantes-chimie et plantes-milieu physique : les dates de campagne correspondent aux observations botaniques. Les dosages des éléments chimiques sont réalisés par spectrophotométrie pour les nutriments et absorption atomique à la flamme pour les cations métalliques.

\section{Analyse de la végétation}

Le but est d'analyser l'information contenue dans les relevés issus de l'application de «MEV » sur une rivière soumise à de fortes pressions anthropiques, en essayant de dégager des caractères synthétiques généraux. L'interprétation pour évaluer la réponse des structures végétales aux variations du milieu est la conjonction d'informations étudiées de manière complémentaire :

\section{Indices de structure}

Ces indices de structure ou de diversité sont issus de la théorie de l'information (PIELOU, 1975) : d'approche très globale, leur but est d'étudier les communautés, en les caractérisant par leur diversité. La littérature contient de nombreux exemples des domaines biologiques les plus divers: les auteurs se servent de ces indices pour formaliser la variété du monde vivant, des structures génomiques aux structures écosystémiques (FRANC et GOUYON, 1997). Dans une problématique similaire de gestion des rivières, BEISEL et al. (1998) ont pu éprouver, par l'utilisation d'indices de structure, l'échantillonnage de communautés macrobenthiques selon le protocole IBGN. DAGET (1980) indique des niveaux complémentaires d'indice de diversité. A partir de nos relevés de végétation des stations de $50 \mathrm{~m}$, nous utiliserons :

- la richesse spécifique, ou nombre d'espèces rencontrées dans le relevé ;

- la diversité informatique, par l'utilisation de l'indice de Shannon, et le calcul d'équitabilité associé.

Pour un échantillonnage suffisant, l'indice de Shannon suit une distribution normale (TAYLOR, 1978, in MAGURRAN, 1988). Cela permet l'utilisation des statistiques paramétriques (ANOVA), méthodes puissantes pour comparer des jeux d'échantillons (SHAW, 1993). Les comparaisons des autres indices utilisent des tests non paramétriques, test de Wilkoxon (POTVIN et ROFF, 1993 ; RECKHOW et al., 1990). 
Indices macrophytiques du Groupement d'intérêt Scientifique "Macrophytes des Eaux Continentales " ou "Indices Biologiques Macrophytiques » : "IBM »

L'Indice Macrophytique du G.I.S. «Macrophytes des Eaux Continentales » ( "IBM ») se présente sous la forme de calcul à partir de la cote spécifique de chacune des plantes du relevé, (HAURY et al., 1996b). Cet indice macrophytique, tel que proposé par HAURY, n'a jamais été utilisé sur la rivière Rance et n'a pas encore fait l'objet d'essai dans des secteurs régulés comme les avals de barrages hydroélectriques. II s'agit là d'une étape de validation et d'amélioration de ces indices sur une rivière très perturbée dès ses sources.

Cet indice peut se calculer selon différentes modalités (6 en tout). Devant la diversité des déclinaisons de l'IBM, nous en avons sélectionné deux : nous avons choisi de tenir compte des calculs de l'IBM en présence-absence des plantes plutôt que de l'estimation de leur recouvrement. En effet, cette valeur d'abondance est en lien avec les capacités d'extension et de multiplication des individus, très variables selon les taxons que l'on considère, plus que de leur valeur bio-indicatrice (DANIEL, 1998). NEWBOLD et HOLMES (1987) n'ont pas non plus retenu cette option, qu'ils avaient initialement envisagée. Dans le premier mode de calcul choisi, nous sélectionnons dans la liste du relevé les espèces indiquées comme sténoèces : IBM « espèces sténoèces ». Pour le second, nous calculons l'IBM « toutes espèces », incluant toutes les espèces à caractères sténoèces du calcul précédent et les autres espèces indicatrices de la liste donnée par HAURY et al. (1996b). Une plus grande sensibilité de l'IBM « espèces sténoèces " doit être attendue par rapport à l'autre, moins sélectif, incluant des espèces a priori moins bio-indicatrices.

\section{Types écologiques et groupes taxonomiques}

II est intéressant pour comparer le fonctionnement des stations de distinguer les représentations des types écologiques et groupes taxonomiques. A partir des relevés, on considère les pourcentages de recouvrement des algues, bryophytes et plantes vasculaires. Une distinction est faite pour ces dernières entre les types écologiques rencontrés, hydrophytes totalement immergées et hydrophytes partiellement immergées. Ces hydrophytes partielles sont parfois abusivement désignées solıs l'intitulé " hélophytes": ce terme est très restrictif, car il désigne au sens de RAUNKIAER (1905), les plantes dont les bourgeons persistants sont protégés de l'hiver par l'eau ou un sol imbibé. Cela permet de tenir compte des différences d'architecture des stations. Ce classement regroupe des notions de classification taxonomique et écologique, ce qui est justifié doublement par une réalité fonctionnelle au sein de l'écosystème et une commodité pour l'échantillonnage. Par station, les résultats seront exprimés en pourcentage de recouvrement de chacun de ces quatre groupes et types (algues, bryophytes, hydrophytes, hydrophytes partielles), ainsi que du pourcentage non végétalisé du lit (" sol nu »), donnant un aperçu structural de la rivière.

\section{RÉSULTATS}

\section{Le contexte physico-chimique de la Rance}

La Figure 2 donne pour les paramètres clefs mesurés en juin, juillet et septembre leur évolution moyenne sur le linéaire, des sources au canal d'llle-et-Rance. Trois tendances, parfois imbriquées, dans ces variations des paramètres chimiques apparaissent. A partir des connaissances du milieu, elles traduisent trois perturbations majeures : 

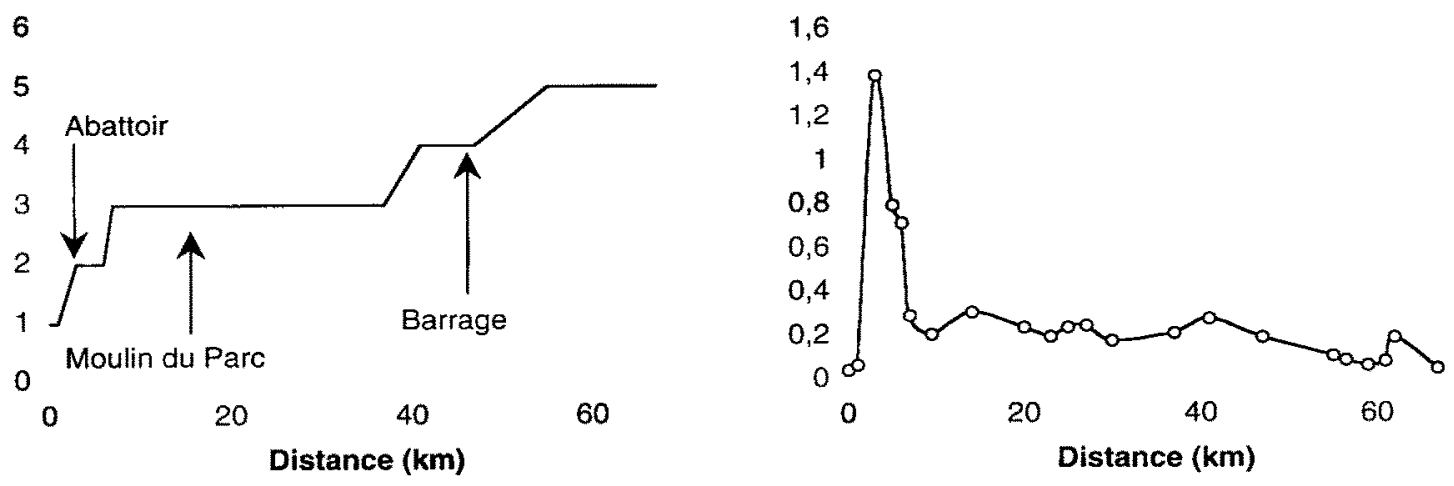

CONDUCTIVITE $\left(\mu \mathrm{S} / \mathrm{cm}^{2}\right)$
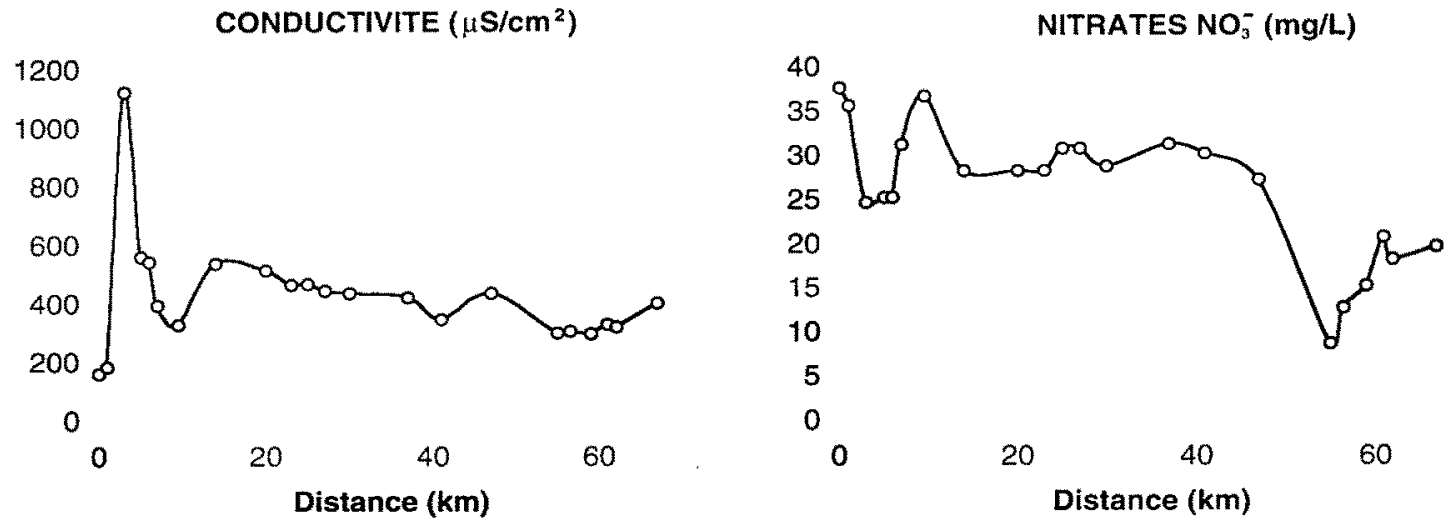

NITRITES $\mathrm{NO}_{2}^{-}(\mathrm{mg} / \mathrm{L})$

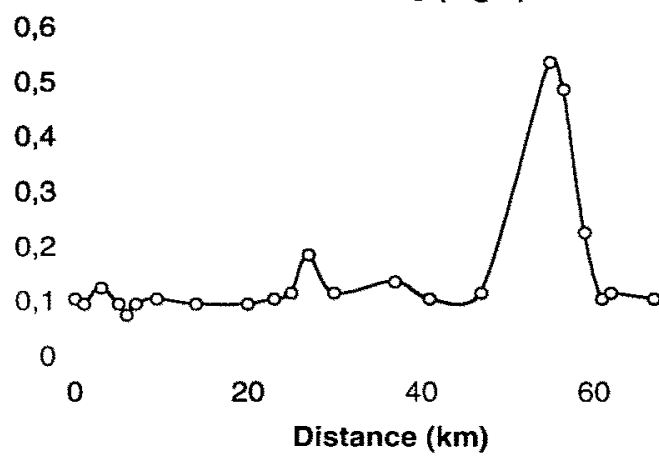

AMMONIAC $\mathrm{NH}_{4}^{+}$(mg/L)

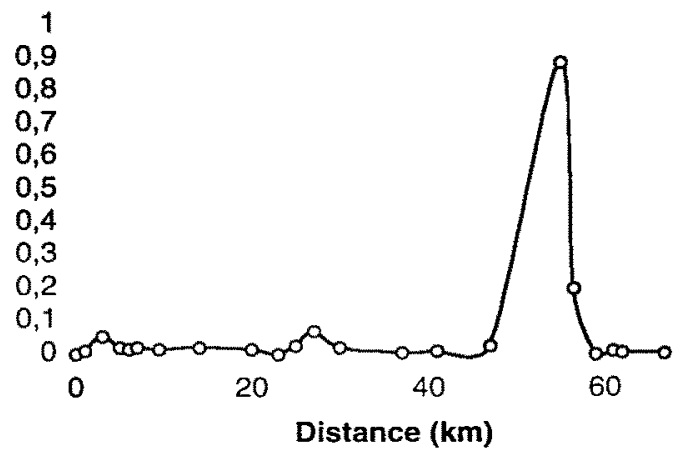

\section{Figure 2}

Mesures physico-chimiques moyennes ( 2 campagnes) en 22 points répartis sur le linéaire du cours d'eau, des sources (ordre de drainage 1) à $15 \mathrm{~km}$ à l'aval du barrage (ordre de drainage 5 ).

\section{Figure 2}

Physico-chemistry (mean of 2 dates) along 22 points of the River Rance, from stream order 1 (" abattoir ": industrial slaughterhouse), stream order 3 (" Moulin du Parc ": intensive agricultural area), to stream order 5 (" barrage ": hydro-electric dam), $15 \mathrm{~km}$ downstream from the impoundment. 
- un premier profil apparaît pour les phosphates et la conductivité (corrélation : 0,90 ), offre un pic de concentration peu après les sources de Collinèe et une atténuation progressive au fil de l'eau, ces paramètres sont également fortement corrélés (de 0,75 à 0,98 ) au sodium, potassium, et chlorures. La position très localisée du pic correspond à une pollution ponctuelle après le rejet de la lagune des abattoirs de Collinée, ordre de drainage 2 (avec des valeurs de conductivité pouvant avoir des maxima au-delà de $\left.2000 \mu \mathrm{S} / \mathrm{cm}^{2}\right)$;

- un second profil présente un plateau important à partir de l'ordre de drainage 3 de la Rance, au niveau du Moulin du Parc, la teneur en nitrates augmente fortement et se stabilise. Ce plateau se forme aussi pour la conductivité, pour les chlorures et pour le potassium (corrélations de 0,77 à 0,995 avec les nitrates). Les teneurs élevées en nitrates de la Rance dès sa source, leur augmentation au Moulin du Parc, leur maintien jusqu'au barrage, la reprise lorsqu'on s'en éloigne, indiquent une pollution agricole diffuse pour toute la Rance. Cela correspond bien au contexte d'agriculture et d'élevage intensifs du bassin. L'atténuation en nitrates à partir de l'ordre de drainage 2 de la Rance est due au passage de la rivière dans la lagune des abattoirs;

- un troisième profil est caractérisé par un pic important de nitrites et d'azote ammoniacal à l'aval de Rophémel (corrélation de 0,83). L'effet du barrage se fait ressentir sur des paramètres classiques des rejets de lac, avec une augmentation de l'azote nitreux et ammoniacal couplée à une récupération rapide (en moins d'un kilomètre à l'aval).

\section{Variations des communautés végétales de la Rance}

\section{Les indices de structure}

\section{La richesse spécifique}

La richesse spécifique croît d'amont en aval (Figure 3). L'effet du barrage se manifeste par une augmentation du nombre d'espèces. Sur tout le linéaire du cours d'eau des variations stationnelles sont observables. La richesse spécifique est plus forte en début d'été qu'en fin, sauf au pied de barrage et dans le début du chenal d'éclusée de Rophémel, où elle se maintient à un niveau élevé aux deux périodes, marquant de manière nette l'effet du barrage en septembre.

\section{Les indices de diversité}

\section{Lindice de Shannon}

A partir du calcul de l'indice de diversité de Shannon, la variabilité de la richesse spécifique est étudiée. Les secteurs régulés à l'aval de Rophémel (maximum $H^{\prime}=0,771$ ) et les secteurs non régulés de l'amont (maximum $H^{\prime}=0,978$ ) semblent être différents du point de vue de leur végétation. Par l'étude statistique de la variance de l'indice de Shannon, ces secteurs sont comparés. Disposant de résultats du début et de la fin de l'été, il est aussi possible de voir si l'effet saisonnier est le même à l'amont qu'à l'aval du barrage. Le signe de la différence saisonnière est négatif à l'amont, positif à l'aval, de valeur absolue identique (moyennes $=0,04$ ). Pour tester les variations spatio-temporelles, une ANOVA est réalisée entre l'amont du barrage et l'aval, aux deux dates, ainsi que pour chaque secteur.

L'hypothèse d'égalité des moyennes de l'indice de Shannon est rejetée pour la comparaison amont-aval. Pour les secteurs de l'amont du barrage, avec une probabilité de 0,88 , l'hypothèse d'égalité entre juillet et septembre peut être acceptée : une rupture due au barrage est confirmée.

On a un lien entre la structure végétale de début et fin d'été à l'amont. Cette relation intersaisons ne se retrouve pas dans les secteurs régulés. 

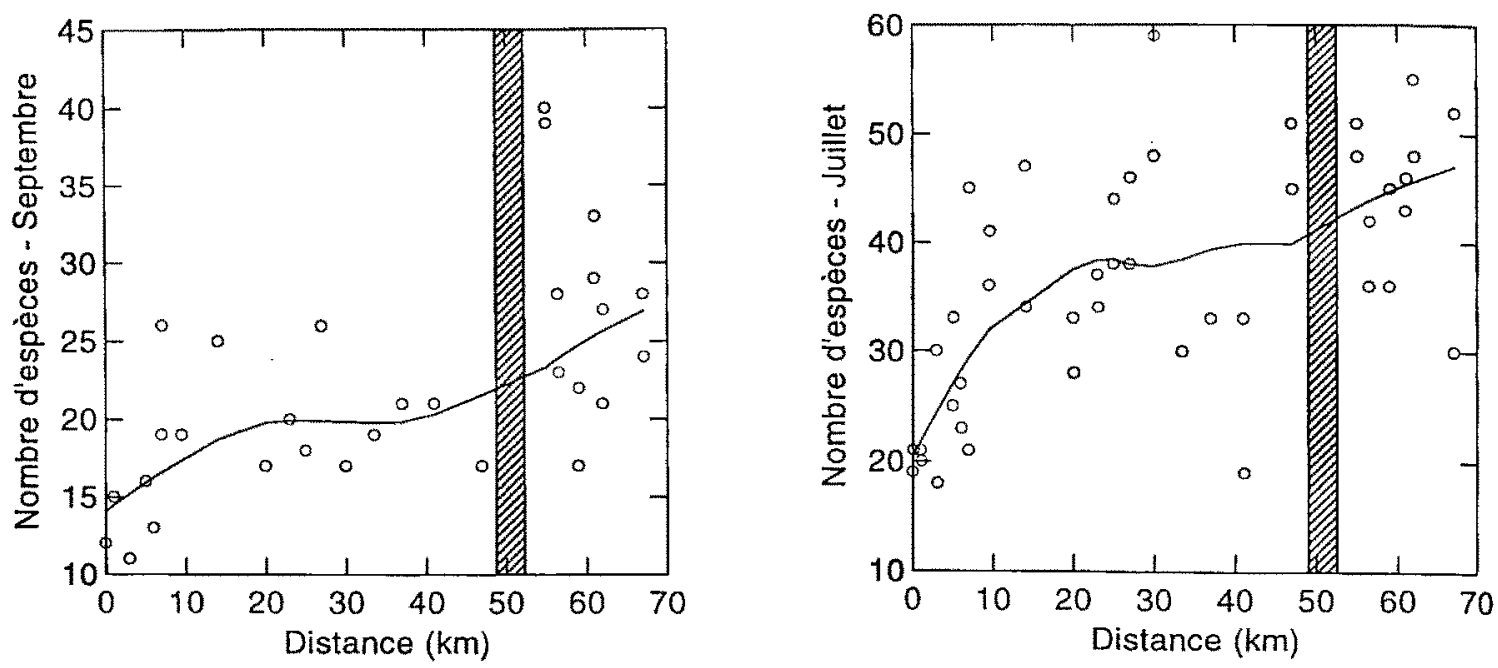

\section{Figure 3}

Evolution de la richesse spécifique en plantes aquatiques aux 2 campagnes estivales des sources à l'aval lointain du barrage (15 km). Courbe de lissage selon la méthode de Lowless (tension 0,5; WILKINSON et HILL, 1994). La zone ombrée représente la position du lac de barrage.

\section{Figure 3}

Specific richness along the River Rance in July and September from sources to $15 \mathrm{~km}$ downstream from the dam. Lowless's curve (tension : 0.5 , WILKINSON and HILL, 1994). The shaded area represents the position of the hydro-electric dam's lake on the longitudinal gradient.

\section{L'équitabilité}

Les informations obtenues avec la richesse et lindice de diversité sont complétées par les variations de l'équilibre de répartition des taxons au sein des stations. Les valeurs d'équitabilité et la différence d'équitabilité permettent également d'appréhender la dynamique saisonnière.

Ainsi, en terme de structure de végétation, l'existence de secteurs particuliers est confirmée, telle que pour la richesse spécifique et l'indice de Shannon à l'aval du barrage. La Figure 4 donne des valeurs d'équitabilité aux deux dates et la différence " juillet " moins "septembre ", des sources jusqu'à l'aval lointain de Rophémel $(15 \mathrm{~km})$. Ce paramètre fluctue sur le gradient longitudinal, mais les valeurs d'équitabilité de juillet et septembre semblent suivre une même tendance, sauf dans quatre secteurs, représentés en grisé : l'aval des abattoirs de Collinée, le secteur d'augmentation des nitrates (au Moulin du Parc, passage de la Rance à l'ordre de drainage 3), le chenal de Rophémel et, de manière moins tranchée, l'aval de la station d'épuration (STEP) de Caulnes.

La différence « juillet » moins « septembre " montre une inversion de l'équitabilité (partie positive de la différence entre juillet et septembre) dans ces quatre secteurs. Les taxons y sont répartis de façon moins homogène à la fin de l'été que sur les secteurs non repérés comme perturbés. L'amplitude de la différence est une indication de l'importance de la variation. 


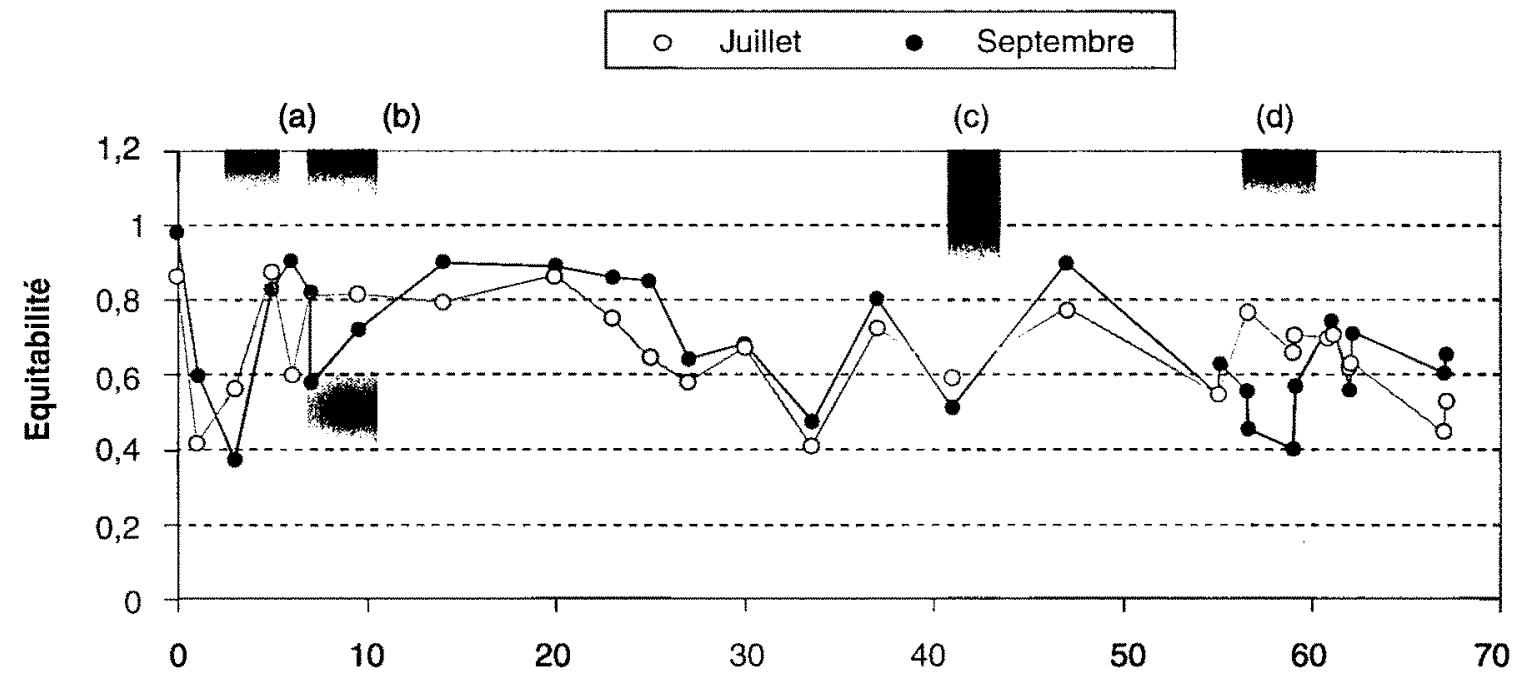

(a) (b)

(c)

(d)

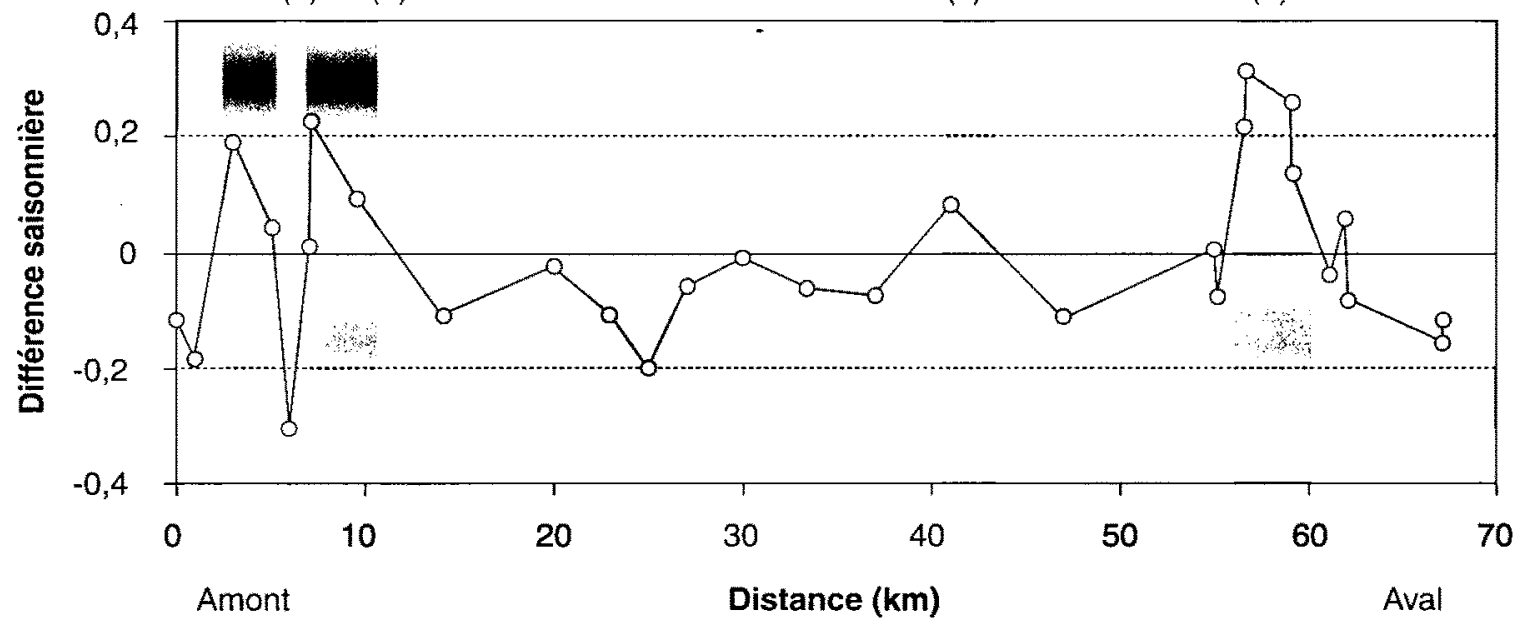

Figure 4

Evolution du calcul d'équitabilité aux deux dates sur le linéaire de la Rance ainsi que de leur différence saisonnière ; les points de pollution sont reportés sur le linéaire ; secteur ombré (a) : aval des abattoirs ; (b) : le Moulin du Parc, agriculture intensive ; (c) : STEP ; (d) : chenal du barrage de Rophémel.

Figure 4

Evenness in July and September and their seasonal differences along the River Rance ; pollution points are reported ; shaded area (a) : industrial slaughterhouse ("abattoirs") ; (b) : nitrate increase due to intensive agriculture - see Figure 2 - («Moulin du Parc ») ; (c) : plant station of the town of Caulnes ("STEP ") ; (d) : downstream channel of the hydro-electric dam of Rophémel (" chenal ").

La contribution de ces variations respectives à ces quatre points de perturbations doit pouvoir être testée. Le test de Wilcoxon permet de connaître la probabilité associée à l'acceptation de l'égalité de ces valeurs d'équitabilité entre le début et la fin de l'été (Figure 5). 
En prenant l'ensemble des $67 \mathrm{~km}$ de la Rance et les 30 couples de valeurs d'équitabilité (60 points), la probabilité d'accepter que la campagne de juillet soit semblable à celle de septembre est de 0,54. En ôtant une à une les valeurs des quatre secteurs repérés précédemment, sont obtenues les probabilités d'acceptation reportées dans la Figure 5. La probabilité d'acceptation inférieure à 0,001 s'obtient en ôtant tous les secteurs perturbés (40 points de comparaison). Le seul secteur du chenal exclu permet aussi d'accepter l'hypothèse à un seuil de 0,05 . De manière moins tranchée, les secteurs subissant des pollutions chimiques, industrielles, agricoles et rejets de STEP diminuent respectivement les probabilités à $0,32,0,27$ et $0,45(56,54$ et 58 points de comparaison).

Les stations sans perturbation apparente présentent une augmentation d'équitabilité de juillet à septembre (Figure 4). Statistiquement leurs valeurs aux deux dates sont en lien (Figure 5), établissant la tendance du comportement stable de la végétation aquatique dans les parties sans perturbation ; l'équitabilité y augmente en fin d'été. Les valeurs dans les quatre secteurs perturbés diminuent en septembre. La contribution du chenal est importante par rapport aux autres perturbations (Figures 3 et 4).

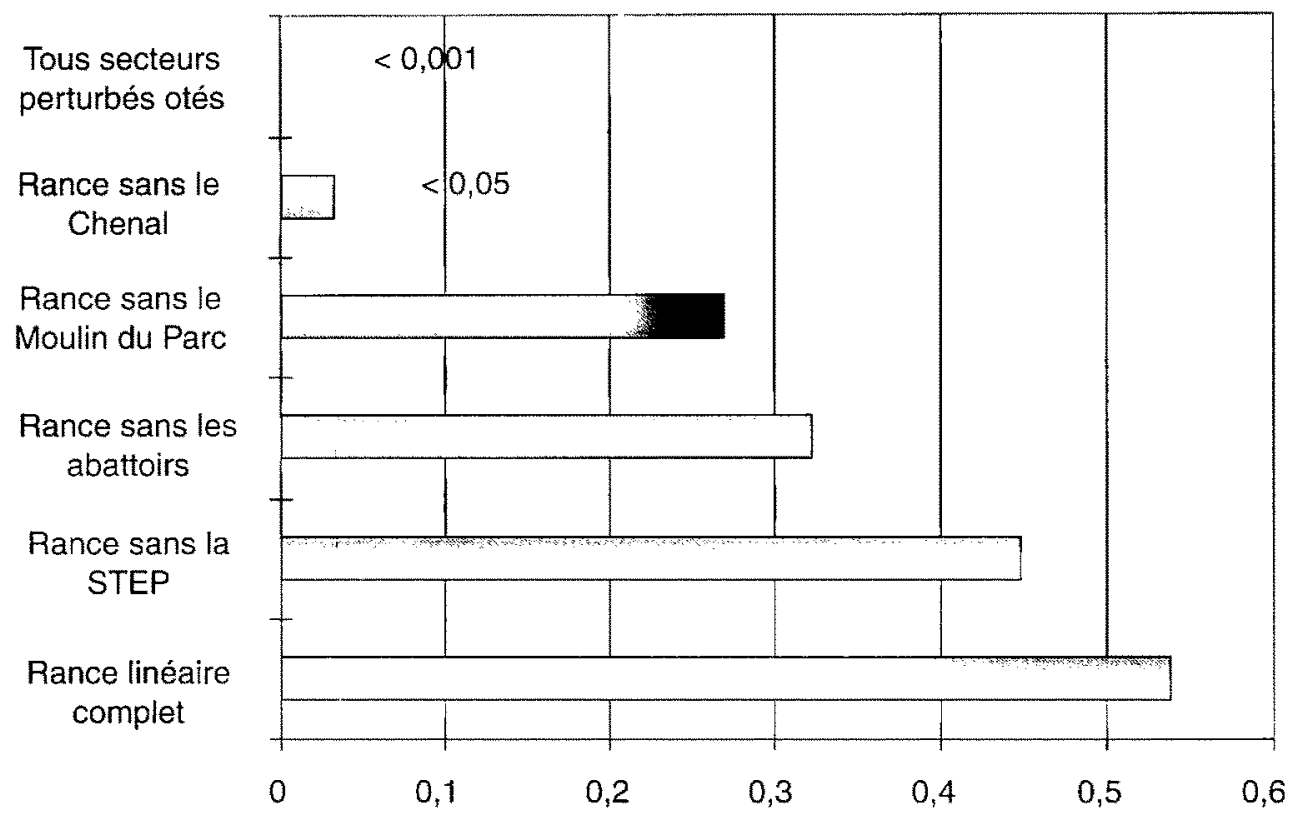

Probabilité d'acceptation de l'égalité entre les deux campagnes

\section{Figure 5}

Test de Wilcoxon comparant les valeurs d'équitabilité entre juillet et septembre : les probabilités d'accepter une relation entre la campagne de début et de fin d'été sont données pour toutes les valeurs de la Rance puis en ôtant les secteurs perturbés - voir texte et Figure 4 -.

\section{Figure 5}

Wilcoxon test to compare evenness of July and of September in the survey of the whole River Rance (first case) ; the River Rance without the plant station of the town of Caulnes ("STEP ") ; the River Rance without the industrial slaughterhouse area (" abattoirs"); the River Rance without the area of "Moulin du Parc "- see Figure 4 for details - ; the River Rance without the downstream channel of the hydro-electric dam (probability of accepting the equality between the periods $<0.05$ ) ; the River Rance without all those perturbed stretches (last case : probability of accepting the equality between the periods $<0.001$ ). 


\section{Variation des indices macrophytiques sur le linéaire de la Rance}

\section{Espèces contributives}

Avant de détailler les résultats des notes obtenues sur le linéaire du cours d'eau, il est important de vérifier le nombre d'espèces qui a contribué au calcul de l'indice. La liste de l'IBM ne comporte pas forcément toutes les espèces des relevés et a fortiori en utilisant l'IBM « espèces sténoèces ». Pour comparaison (Tableau 1), les indices anglo-saxons, pour l'indice trophique de NEWBOLD et HOLMES (1987), donnent de 5 à 24 espèces contributives par relevé, et avec l'indice de HARDING de 7 à 31 (HARDING, 1978 in STANDING COMMITTEE OF ANALYSTS, 1987) duquel est issu l'IBM (HAURY et al., 1996b), ce qui explique les similitudes observées dans ce cas.

\section{Tableau I}

Valeurs minimales, maximales et moyennes de 4 indices macrophytiques et le nombre d'espèces qui contribuent au calcul pour les 44 relevés de juillet.

\section{Table I}

Scores of 4 macrophytes indexes (minimum, maximum and mean value) and the number of species contributing to each score for the $\mathbf{4 4}$ survey of July.

\begin{tabular}{|l|c|c|c|c|c|c|c|c|}
\hline & IBM & $\mathrm{nb} s p$ & $\begin{array}{c}\text { IBM } \\
\text { sténoèces }\end{array}$ & $\mathrm{nb} \mathrm{sp}$ & $\begin{array}{c}\text { Newbold } \\
\text { \& Holmes }\end{array}$ & $\mathrm{nb} \mathrm{sp}$ & Harding & $\mathrm{nb} \mathrm{sp}$ \\
\hline $\min$ & 4,4 & 8 & 4,4 & 3 & 50,3 & 4 & 3,7 & 7 \\
\hline max & 5,8 & 31 & 7,3 & 20 & 99,3 & 24 & 6,4 & 31 \\
\hline moy & 5,0 & 19 & 5,3 & 11 & 79,0 & 12 & 4,9 & 18 \\
\hline
\end{tabular}

\section{Valeurs de l'IBM}

L'amplitude de variation est plus grande pour l'IBM « espèces sténoèces " (de 4,4 à $7,3)$ que pour I'IBM «toutes espèces» $(4,4$ à 5,8). L'amplitude de l'IBM "espèces sténoèces" est très proche de celle de l'indice d'Harding. Cependant pour l'indice "sténoèces", les valeurs extrêmes sont obtenues pour un nombre d'espèces contributives faible, respectivement pour les valeurs précitées, 5 et 3 . La note maximale est obtenue à l'aval de l'abattoir $(7,3)$, mais ce nombre est calculé à partir de la cote de trois espèces seulement. L'une d'entre elles, Ranunculus omiophyllus est indicatrice de très bonne qualité d'eau. Ainsi, le choix des valeurs présentées dans cette étude se porte vers l'indice «toutes espèces", avec 8 taxons pour le calcul présentant le moins d'espèces. Les deux indices anglo-saxons, s'ils ont un nombre d'espèces contributives comparable, offrent une amplitude de notes plus grande que l'IBM " toutes espèces » (Tableau 1). Par contre les stations correspondant aux valeurs extrêmes concordent entre I'IBM “ toutes espèces" et les deux indices de Newbold \& Holmes et d'Harding. La Figure 6 présente donc les résultats aux deux dates sur le linéaire de la Rance (selon la distance aux sources) des valeurs obtenues par le calcul IBM « toutes espèces ". De par l'homogénéité des notes observée sur tout le linéaire, une relation avec les secteurs repérés comme perturbés n'est pas envisageable. Pour comprendre le manque de réponse de l'indice IBM sur cette rivière, sur la Figure 7 est reportée la répartition des taxons indicateurs vus sur la Rance en fonction de leurs cotes dans l'IBM. Pour les deux dates, la majeure partie des taxons contribuant à la note IBM est répartie dans les classes de notes $4,5,6$ ou 7 , avec deux courbes en cloche, centrées sur la moyenne. Un petit décalage est noté entre les deux dates: plus de valeur 4 et moins de 6 , 7 et 8 en septembre, soit un effet de baisse de la note IBM à la fin de l'été. Le nombre de taxons indicateurs impliqués est constant aux deux dates. Une dégradation de la note de l'indice serait donc observable entre juillet et septembre, ces calculs aboutissant simplement à une légère sensibilité saisonnière. Les structures de végétation des secteurs 
marquants à ces deux dates sont intéressantes à étudier à l'aide des recouvrements des différents types écologiques et groupes taxonomiques rencontrés. Le but est de confirmer cet effet saisonnier qui est pressenti par l'IBM et d'observer la réponse végétale aux perturbations identifiées par les indices de structure et le contrôle de la qualité de l'eau préalables.
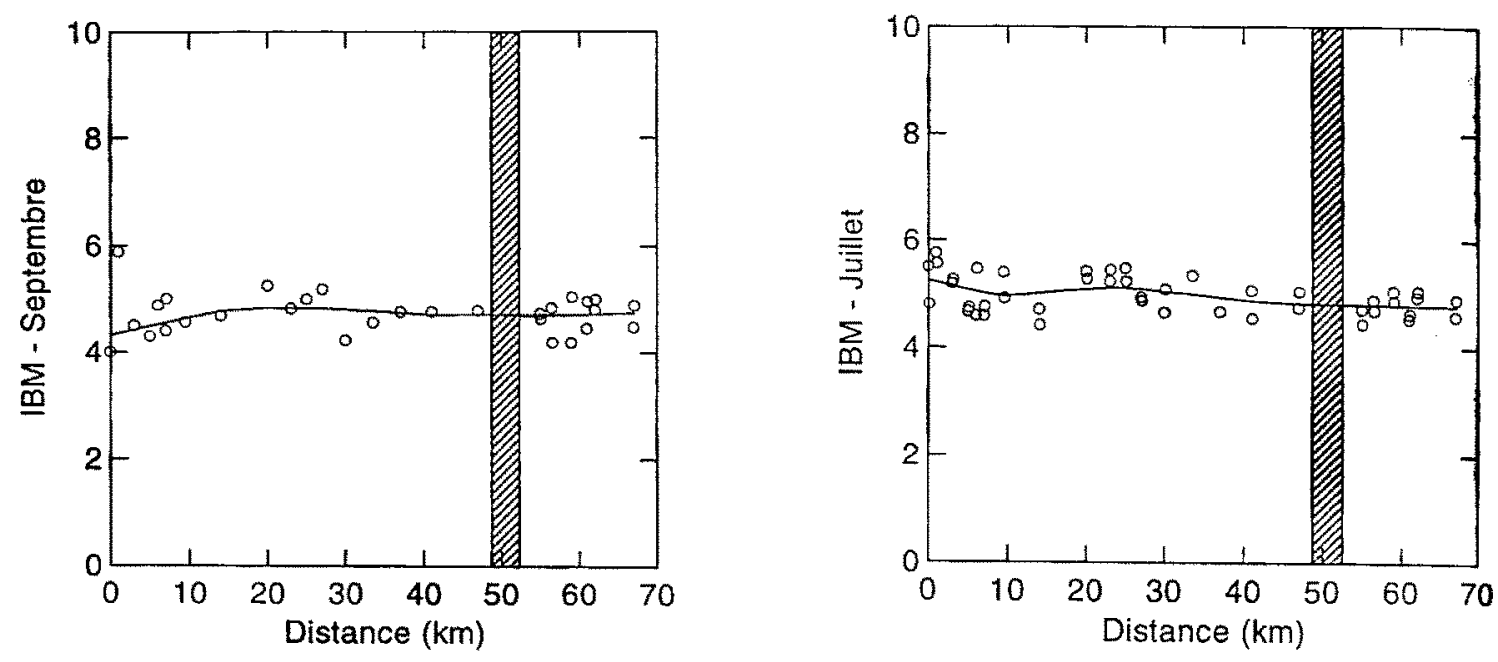

Figure 6

Indice Biologique Macrophytique «IBM toutes espèces » (HAURY et al., 1996b) en juillet et septembre sur les $67 \mathrm{~km}$ de linéaire de rivière. Courbe de lissage selon la méthode de Lowless (tension 0,5; WILKINSON et HILL, 1994). La zone ombrée représente la position du lac de barrage.

Figure 6

Macrophyte index “IBM " (HAURY et al., 1996b) in July and September along the $67 \mathrm{~km}$ of the River Rance. Lowless's curve (tension : 0.5, WILKINSON et HILL, 1994). The shaded area represents the position of the hydro-electric dam's lake on the longitudinal gradient.

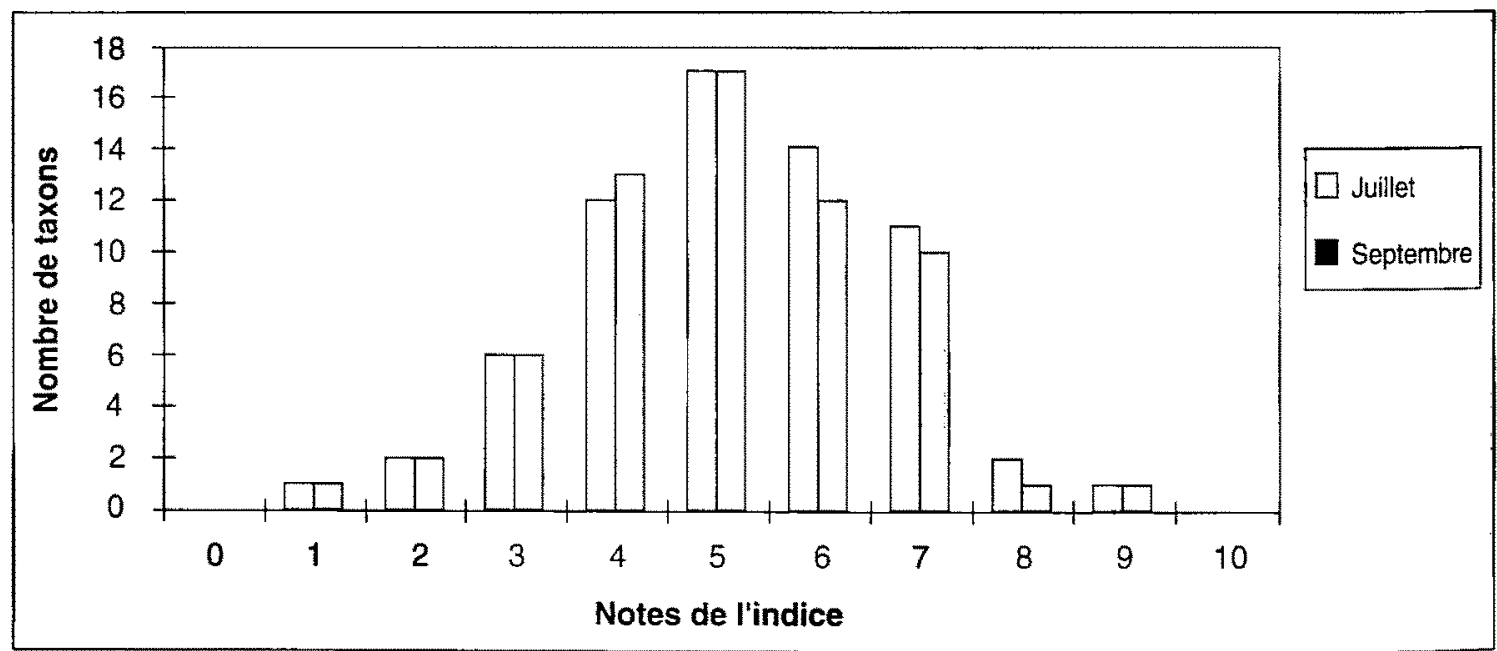

Figure 7

Répartition du nombre de taxons selon leur cote dans l'indice. « IBM toutes espèces " (HAURY et al., 1996b) pour l'ensemble des relevés de juillet et septembre.

\section{Figure 7}

Distribution of number of taxa according to their score in the macrophyte index "IBM " (HAURY et al., 1996b) for the survey of July and September. 
Types écologiques et groupes taxonomiques de la Rance

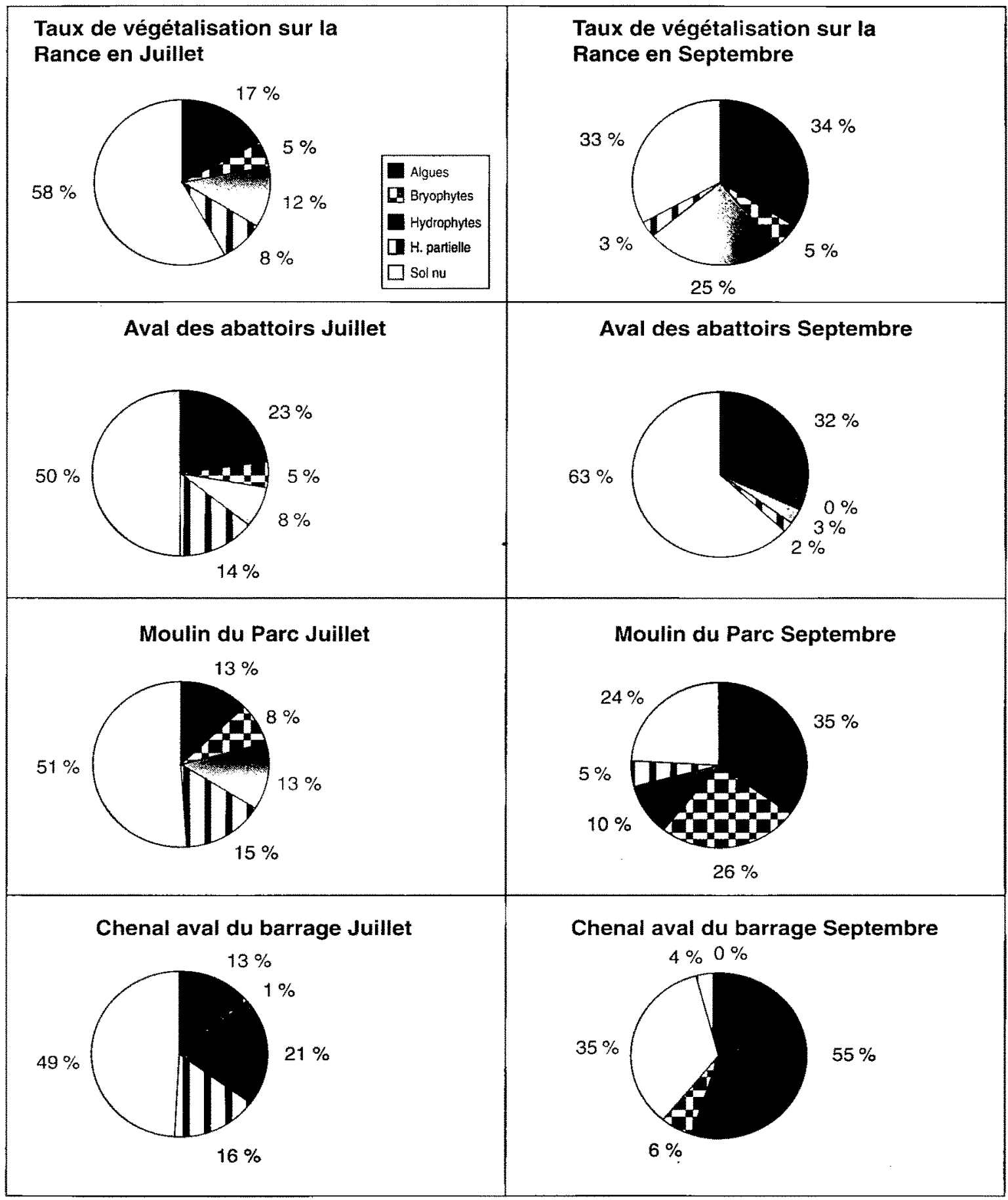

\section{Figure 8}

Pourcentages de recouvrement moyen des groupes et types biologiques, de la part non végétalisée, en juillet et septembre sur la Rance et dans les secteurs perturbés principaux.

\section{Figure 8}

Percent of mean cover of biological groups and types and bare substrate (" sol nu $")$ in July and September, for the whole River Rance and the 3 main perturbed areas (" abattoirs": industrial slaughterhouse ; "Moulin du Parc ": localised trophic enrichment - see Figure 2 - ; « chenal ": downstream channel of the hydro-electric dam). 
La Figure 8 donne les pourcentages de recouvrements moyens des types et groupes rencontrés et la surface non végétalisée (sol nu) des stations. Les 8 représentations correspondent aux relevés de juillet et septembre sur l'ensemble de la Rance et ceux des stations des trois secteurs perturbés principaux. L'aval de la STEP ne présente ni variations physico-chimiques, ni variations marquées des indices de structure ; il est donc inclus dans l'ensemble de la Rance.

Les trois secteurs repérés sont toujours plus végétalisés que l'ensemble du cours d'eau (juillet : respectivement $50 \%$ de sol nu contre $58 \%$ ), sauf l'aval des abattoirs en septembre. Les recouvrements augmentent entre juillet et septembre sauf pour l'aval des abattoirs. Les algues sont moins importantes dans le secteur du Moulin du Parc ainsi que dans le chenal en juillet. Elles augmentent fortement à la fin de l'été, surtout dans le chenal de Rophémel (13 à $55 \%$ ). Les bryophytes qui ne subissent pas globalement de variations à l'échelle de la rivière entre les deux dates, disparaissent de l'aval des abattoirs, triplent au Moulin du Parc où elles recouvrent plus du quart du secteur, sextuplent dans le chenal. Au cours de l'été, les plantes vasculaires hydrophytes doublent de recouvrement sur la Rance. Leur progression dans le chenal est plus faible mais atteint le plus fort recouvrement $(35 \%)$. Elles régressent à l'aval des abattoirs et dans le secteur du Moulin du Parc où elles étaient moins représentées en juillet qu'ailleurs. Les hydrophytes partielles diminuent globalement au cours de l'été et sont favorisées dans les trois secteurs perturbés surtout en juillet.

\section{DISCUSSION}

\section{Variations spatiales}

Sur la Rance, les secteurs dits «perturbés " sont plus végétalisés que les autres. L'échantillonnage stratifié par rapport à ce gradient a permis de mettre en évidence des secteurs diversement pollués : une réponse des communautés végétales est observée pour des perturbations fortes au sein de cette variation longitudinale. Les barrages sont des éléments de rupture de cette continuité (WARD et STANFORD, 1983a). Dans cette étude, la perturbation physique liée au barrage est réduite pendant les deux mois où l'utilisation principale de Rophémel est l'adduction d'eau potable. Cette quasi absence d'éclusée et un débit réservé faible $\left(0,09 \mathrm{~m}^{3} / \mathrm{s}\right)$, offrent aux différents groupes de végétaux, la possibilité de coloniser l'espace rendu disponible par l'arrêt des contraintes hydrauliques. Cela donne une physionomie particulière au secteur aval du barrage, que décrivent les valeurs de recouvrements des types et groupes biologiques.

EVERITT et BURKHOLDER (1991) s'affranchissent de cette variation longitudinale en comparant la végétation de secteurs d'ordre de drainage constant (ordre 2 et 3 ). Ainsi, les différences de recouvrement entre les types écologiques et groupes taxonomiques à l'aval de l'abattoir (ordre 2) et dans le chenal du barrage (ordre 5) pourraient être attribuées au gradient longitudinal. L'équitabilité et les différences d'équitabilité montrent que tous les secteurs perturbés ont un fonctionnement comparable pour l'équilibre de la répartition des taxons, qui est inversé par rapport aux secteurs non perturbés. Si un antagonisme apparaît pour les groupes taxonomiques des zones perturbées au long de la Rance, leur dynamique y est semblable. WARD et STANFORD (1983a) montrent qu'en fonction de la position du barrage, on peut observer des diminutions (barrage en situation amont et médiane) ou des gains (situations avales) de diversité biologique par rapport aux secteurs de tête de bassin. Les hydrophytes diminuent d'abondance pour un barrage en situation médiane, mais c'est l'inverse pour une situation avale. A l'échelle de la Rance, le barrage de Rophémel correspondrait à une position avale de WARD et STANFORD, et les communautés végétales observées tendent aussi dans ce sens. La forte diversité à l'aval du barrage va également dans le sens d'un concept repris par WARD et STANFORD 
(1983b) pour expliquer ces phénomènes par rapport à l'c intermediate disturbance hypothesis " de CONNELL (1978), niveau de perturbation moyen qui permet une diversité maximale. Dans son étude de la dynamique des communautés algales sur la Durance, FAYOLLE (1998) a montré une réaction quantitative prépondérante par rapport à la richesse taxonomique concernant l'épilithon. A l'aval du barrage de Rophémel, les abondances de recouvrement ainsi que les richesses spécifiques des macrophytes sont amplifiées par rapport aux autres secteurs.

\section{Variations temporelles}

L'étude la végétation nécessite de se placer dans les périodes d'optimum de développement liées aux variations saisonnières (DAWSON, 1978 ; DAWSON et al., 1978). Un protocole d'étude avec deux campagnes estivales est un minimum pour acquérir des données, une liste d'espèces la plus complète (HAURY et al., 1998) est recueillie.

Cela permet aussi d'appréhender des aspects de dynamique de la végétation, plus riches d'enseignement qu'un instantané pour percevoir le fonctionnement de l'écosystème. EVERITT et BURKHOLDER (1991), sur des rivières granitiques américaines d'ordre 2 et 3 , montrent que cette dynamique des communautés est sous l'influence d'interactions compétitives entre les macrophytes. C'est une hypothèse avancée par DANIEL et HAURY (1996) à l'aval des piscicultures du Scorff (Basse-Bretagne). MC CREARY (1991) donne les compétitions entre espèces comme explication des structures des communautés macrophytiques, considérées comme hypothèse partielle par rapport à l'effet du milieu pour CHAMBERS et PREPAS (1990). Dans le cas des secteurs perturbés sur le linéaire de la Rance, l'étude des variations estivales est intéressante. Ainsi les différences entre juillet et septembre y sont caractérisées par:

- une diminution de la richesse en espèces sauf à l'aval du barrage ;

- un lien de la diversité entre les deux dates pour l'amont du barrage, pas pour l'aval ;

- des différences de la répartition des taxons dans les secteurs perturbés;

- une baisse globale de la note de l'indice IBM en septembre ;

- des augmentations des recouvrements végétaux pour toute la rivière à l'étiage de septembre.

Des stades proliférants, correspondant à des phénomènes de développement exacerbé (PRYGIEL, 1997), sont atteints dans les secteurs perturbés. Ceci touche, selon les types de perturbations identifiés, des types écologiques et des groupes taxonomiques variés. La réponse végétale entre le début de l'été et l'étiage est observable avec les différents outils d'interprétation mis en oeuvre : les proliférations sont bien sûr repérées au niveau des recouvrements des groupes et types biologiques. EVERITT et BURKHOLDER (1991) mettent en évidence une interaction compétitive entre angiospermes hydrophytes et algues, deux groupes dominants dans les secteurs perturbés de la Rance. MADSEN et ADAMS (1988) dans leurs études des dynamiques saisonnières décrivent des successions entre Cladophora glomerata (macro-algue) et Potamogeton pectinatus (angiosperme hydrophyte).

Les variations saisonnières sont observables également par l'étude des valeurs d'équitabilité, ceci est aussi à relier aux tendances proliférantes. L'étude de cette dynamique saisonnière devient alors une dimension intéressante d'interprétation. II serait 
intéressant de voir comment cela varie à un pas de temps plus restreint et sur des variations inter-annuelles. La diminution globale de la note IBM en septembre viendrait aussi confirmer des problèmes de croissance proliférante d'algues qui ont des cotes souvent moins bonnes.

\section{Apport et amélioration des outils d'interprétation}

\section{Contribution aux Indices Macrophytiques IBM}

DESMARS et HARPER (1998) préconisent l'utilisation de méthodes statistiques telle que l'analyse canonique des correspondances pour mettre en relation la végétation avec ces paramètres du milieu. DANIEL (1998) a utilisé ces méthodes d'ordination pour mettre en place un indice basé sur le calcul à partir de données de végétation et de qualité du milieu, en Bretagne sur le Scorff, dans le cadre de l'impact des piscicultures. Ces auteurs s'accordent pour indiquer qu'il faut prendre en compte un jeu de données important, qui ne peut être cantonné à une simple rivière où à quelques sites, pour mettre au point un indice pertinent.

L'amélioration de l'indice IBM passe par ce type de validation Sur la Rance, sa mise en œuvre est difficile. C'est une rivière où pourtant se localisent une pollution ponctuelle industrielle, une pollution agricole diffuse et une perturbation hydraulique majeure. DANIEL et HAURY (1996) autour de piscicultures du Scorff ont obtenu des résultats semblables, soient des notes allant de 5 à 6,5 sur 10 , sans tendance franche par rapport au niveau de pollution organique observé. Dans d'autres régions de France pour des pollutions de même type, également sans tenir compte de l'abondance des recouvrements, une réponse plus nette a été obtenue (HAURY et PELTRE, 1993). L'utilisation sur la Rance de I'IBM “ toutes espèces " ne favorise certainement pas ce résultat. L'utilisation d'espèces sténoèces est sans doute plus pertinent dans le concept de bio-indication (MURTAUGH, 1996 ; LEGENDRE et LEGENDRE, 1984). Dans le cas présenté, une étape de validation de la liste des sténoèces sur un jeu de données plus grand est à réaliser. Pour l'IBM, fruit de travaux conséquents, on peut trouver une explication intéressante à ce faible niveau de réponse : sur la Rance, les espèces contributives au calcul correspondent pour la plupart à peu de classes de notes centrées sur la moyenne. Ceci évoquerait l'effet d'une flore aquatique banalisée par un système très perturbé dès ses sources. D'autre part, le nombre minimal d'espèces du calcul des indices n'est pas donné dans le protocole. Cette question devra faire l'objet d'un approfondissement. Ce point de discussion est primordial car la diversité biologique est affectée par le niveau de perturbation (COLLINS et al., 1995 ; BORNETTE et AMOROS, 1996). Qu'en sont les conséquences pour les indices macrophytiques ? II n'est pas envisageable de se fier à la seule présence d'une espèce indicatrice de bonne qualité d'eau à l'aval d'un rejet d'abattoir, ce qui est le cas de Ranunculus omiophyllus dans cette étude. II faudrait vérifier les hypothèses qu'elle puisse être tolérante à des pollutions, ou bien liée à une situation amont, plutôt qu'à une bonne qualité d'eau, ce qui est souvent en lien, mais pas sur la Rance. Ce système perturbé dès les sources devient une référence de travail intéressante pour ce type de problème. Ainsi dans le cadre d'un programme d'amélioration des indices, il faut revenir aux listes d'espèces et à l'étude de leur écologie spécifique pour ne pas engendrer ce type d'erreur. Les méthodes statistiques d'ordination sont une possibilité rigoureuse d'y remédier. Actuellement, faute de disposer d'une méthode de calcul des cotes des espèces de l'IBM, linterprétation des problèmes rencontrés sur la Rance ne peut aller plus loin que les hypothèses énoncées.

\section{Outils et perception du fonctionnement en rivière : les structures végétales}

Les autres approches interprétatives menées sur la Rance, indices de structure et étude des groupes taxonomiques et types écologiques ont donné des résultats plus 
probants, permettant d'aborder le fonctionnement des rivières sous des aspects spatio-temporels et de tester les protocoles d'étude des végétaux en rivière. Des études similaires de perturbations affectant la diversité macrophytique ont été réalisées par DYKYJOVA et al. (1985). BEISEL et al. (1998) ont utilisé ces indices de structure pour éprouver l'échantillonnage de communautés macrobenthiques selon le protocole IBGN : cela leur a permis d'envisager des solutions pour optimiser les méthodes de suivi de la qualité biologique des cours d'eau.

L'importance de la variabilité comme outil d'analyse du fonctionnement des écosystèmes a été plusieurs fois soulignée en écologie (BARBAULT, 1992). Elle est indispensable dans la démarche engagée ici sur la Rance. Une valeur d'équitabilité n'est pas utilisable en tant que telle, mais seulement par rapport à une valeur de référence, comme line comparaison saisonnière. Comme le précise JURKO (1986), les conditions d'utilisations des indices de diversité doivent être rigoureusement comparables. A partir de cette étude, les paramètres de standardisation à préconiser pour une confirmation par comparaison inter-annuelle ou pour d'autres rivières, concerneralent des relevés de végétation sur des secteurs de $50 \mathrm{~m}$, en début et fin d'été, selon le protocole d'échantillonnage hiérarchisé de MEV.

Pour ce qui concerne la prise en compte de l'impact du milieu physique sur la végétation, son importance est signalée (DAWSON, 1988 ; HASLAM, 1978) et confirmée par le calcul statistique (DANIEL, 1998 ; DESMARS et HARPER, 1998 ; PADMORE, 1997). Sur la Rance, dans l'approche de comparaison des valeurs d'équitabilité obtenue à partir de la végétation (Figure 5), on constate statistiquement l'importance de l'effet du chenal. L'effet sur l'aval du barrage est très tranché, et correspond aux travaux menés dans ce type de secteurs sur les problèmes d'espèces proliférantes (KHALANSKI et al., 1990 ; GOSSE, 1989). La primauté de l'effet du milieu physique sur les paramètres chimiques n'est pas complètement démontrée, mais mériterait d'être approfondie à l'aide des observations à l'aval des barrages. Notamment au niveau des sélections d'espèces, approfondir les interactions et phénomènes de compétitions et les stratégies de reproduction. Ces secteurs régulés ont été beaucoup étudiés à l'étranger, en écologie générale des cours d'eau (BAXTER, 1977 ; BROOKER, 1981). De par la diversité des sites en France (CROUZET et LÉONARD, 1995), on peut ainsi envisager de choisir des secteurs en fonction des modes de gestion hydrauliques. On aurait ainsi des études en expérimentation naturelle, d'un intérêt d'autant plus fort que de nombreux barrages sont équipés de systèmes de mesures régulières ou en continu de la qualité physico-chimique de l'eau.

\section{CONCLUSION}

Cette étude des changements structuraux, traduisant le fonctionnement et la dynamique des communautés de la végétation aquatique est une piste essentielle pour la gestion des rivières. DEN HARTOG (1978) l'a souligné pour les aspects d'extension spatio-temporelle et de diversité des plantes aquatiques. L'approche développée ici permet un diagnostic facilement applicable, mode de surveillance à préconiser en amont d'une expertise où une acuité taxonomique fine est requise. Si la nécessité de la détermination floristique précise peut être contournée dans un premier temps, les indices de structure et recouvrements des groupes biologiques restent des descripteurs grossiers de l'écosystème. Ces outils ont donné une réponse pour des pollutions très nettes. Mais il faut maintenir un niveau de détermination suffisant, permettant en plus de cette connaissance des caractères fonctionnels, l'amélioration de la démarche de bio-indication qui en découle. On a pu voir que la réponse qualitative d'une liste taxonomique pouvait être intéressante, mais qu'il ne fallait pas négliger les aspects structuraux des communautés, qui correspondent à une autre forme de réponse aux perturbations. L'étude 
de perturbations liées aux régulations a déjà été réalisée pour comparer des réponses biologiques à des paramètres physiques précis : KING et al. (1998) entre poissons et température. L'intensité de réponse des plantes aquatiques est forte dans ces secteurs, on peut envisager qu'ils deviennent des outils de connaissance de l'écologie spécifique et du suivi du fonctionnement des communautés macrophytiques.

\section{REMERCIEMENTS}

Les auteurs remercient Vincent BOUCHAREYCHAS et Valérie LAHILLE pour leur participation à l'acquisition des données, Laurent BEILLARD, Daniel CHICOUENE pour les corrections au manuscrit, ainsi que EDF, le GME-Bretagne, les Agences de l'Eau Loire-Bretagne et Seine-Normandie pour leur soutien financier.

\section{BIBLIOGRAPHIE}

BARBAULT R., 1992. Dynamique des peuplements - Effets de la variabilité à différentes échelles, de l'individu aux systèmes de populations interconnectées. In: AUGER P., BAUDRY J. et FOURNIER F. (Eds), Hiérarchies et échelles en écologie, SCOPE, Cahors, 127-155.

BAXTER R.M., 1977. Environmental effects of dams and impoundments. Annual Review of Ecology and Systematic, 8, 255-283.

BEAUMONT P., 1975. " Hydrology ». In : WHITTON B.A. (ed.), River ecology, Blackwell Scientific Publications, Oxford, 1-38.

BEISEL J.-N., USSEGLIO-POLATERA P., THOMAS S., MORETEAU J.-C., 1998. Influence de l'échantillonnage des communautés macrobentiques sur l'évaluation de la qualité biologique d'un cours d'eau. Annales de Limnologie, 34(4), 445-454.

BERNEZ I., 1999. Végétation macrophytique des cours d'eau régulés. Impacts des aménagements hydroélectriques dans le Massif armoricain. Thèse de l'Ecole Nationale Supérieure Agronomique de Rennes, $127 \mathrm{p}$.

BORNETTE G., AMOROS C., 1996. Disturbance regimes and vegetation dynamics : role of floods in riverine wetlands. Journal of Vegetation Science, 7, 615-622.

BROOKER M.P., 1981. The impact of impoundments on the downstream fisheries and general ecology of rivers. Advances in Applied Biology, 6, 91-152.

CAIRNS J.J., MCCORMICK P.V., NIEDERLEHNER B.R., 1993. A proposed framework for developing indicators of ecosystem health. Hydrobiologia, 263, 1-44.

CHAMBERS P.A., PREPAS E.E., 1990. Competition and coexistence in submerged aquatic plant communities: the effects of species interactions versus abiotic factors. Freshwater Biology, 23, 541-550.

COLLINS S.L., GLENN S.M., GIBSON D.J., 1995. Experimental analysis of intermediate disturbance and initial floristic composition : decoupling causes and effects. Ecology, 76, 486-492.

CONNELL J.H., 1978. Diversity in tropical rain forests and coral reefs. Science, 199, $1302-1310$.

COUILLARD D., LEFEBVRE Y., 1985. Analysis of water quality indices. Journal of Environmental Management, 21, 161-179.

COURTEMANCH D.L., DAVIES S.P., 1987. A coefficient of community loss to assess detrimental change in aquatic communities. Water Research, 21(2), 217-222.

CROUZET P., LÉONARD J., 1995. Les lacs de grands barrages en France et en Europe. Les données de lenvironnement - IFEN(17), 1-5. 
DAGET P., 1980. Le nombre de diversité de Hill, un concept unificateur dans la théorie de la diversité écologique. Acta Oecologica, 1(1), 51-70.

DANIEL H., 1998. Evaluation de la qualité des cours d'eau par la végétation macrophytique. Travail in situ et expérimental dans le Massif armoricain sur les pollutions par les macronutriments. Thèse de l'Ecole Nationale Supérieure Agronomique de Rennes, $150 \mathrm{p}$.

DANIEL H., HAURY J., 1996. Les macrophytes aquatiques: une métrique de l'environnement en rivière. Cybium, 20(3), 129-142.

DAWSON F.H., 1978. The seasonal effects of aquatic plant growth on the flow of water in a stream. EWRS 5th Symp. on Aquatic Weeds, 71-78.

DAWSON F.H., 1988. Water flow and the vegetation of running waters. In: SYMOENS J.J. (ed.), Vegetation of inland waters, Kluwer Academic Publishers, Dordrecht, 283-309.

DAWSON F.H., CASTELLANO E., LADLE M., 1978. Concept of species succession in relation to river vegetation and management. International Vereinigung für Theoretische und Angewandte Limnologie, 20, 1429-1434.

DEN HARTOG C., 1978. Structural and functional aspects of macrophyte-dominated aquatic systems. EWRS 5th Symp. on Aquatic Weeds, 35-41.

DESMARS B.O.L., HARPER D.M., 1998. The aquatic macrophytes of an English lowland river system : assessing response to nutrient enrichment. Hydrobiologia, 384, 75-88.

DYKYJOVA D., KOSANOVA A., HUSAK S., SLADECKOVA A., 1985. Macrophytes and water pollution of the Zlata Stoka (Golden Canal), Trebon Biosphere Reserve, Czechoslovakia. Archiv für Hydrobiologie, 105(1), 31-58.

EVERITT D.T., BURKHOLDER J.M., 1991. Seasonal dynamics of macrophyte communities from a stream flowing over granite flatrock in North Carolina, U.S.A. Hydrobiologia, 222, 159-172.

FAYOLLE S., 1998. Réponses des communautés végétales strictement aquatiques (algues et macrophytes) aux perturbations hydrodynamiques (débits réservés, restitutions) en Durance aménagée (Sud-Est de la France), Thèse de l'Univ. d'Aix-Marseille III, $227 \mathrm{p}$.

FRANC A., GOUYON P.-H., 1997. Information et complexité : question sans réponse. La Recherche, 296, 106-109.

GOSSE P., 1989. Influence des végétaux fixés sur la qualité de l'eau du Vair et du petit Vair en aval de Vittel. ARD E31 E01, EDF-DER, Chatou.

HASLAM S.M., 1978. River plants, Cambridge University Press, Cambridge.

HAURY J., 1985. Etude écologique des macrophytes du Scorff (Bretagne Sud), Thèse de Doc.- Ing., Univ. Rennes I, 96 p.

HAURY J., PELTRE M.-C., 1993. Intérêts et limites des indices macrophytes pour qualifier la mésologie et la physico-chimie des cours d'eau : exemples armoricains, picards et lorrains. Annales de Limnologie, 29, 239-253.

HAURY J., BERNEZ I., LAHILLE V., 1996a. Influence de la retenue de Rabodanges sur les peuplements macrophytiques de l'Orne. 1er Colloque interceltique d'Hydrologie et de Gestion des Eaux, Rennes (France), $470 \mathrm{p}$.

haury J., PElTRE M.-C., MUller S., TRÉmolières M., BARBE J., DutARTRE A., GUERLESQUIN M., 1996b. Des indices macrophytiques pour estimer la qualité des cours d'eau français: premières propositions. Ecologie, 27(4), 233-244.

HAURY J., JAFFRÉ M., DUTARTRE A., PELTRE M.-C., BARBE J., TRÉMOLIĖRES M., GUERLESQUIN M., MÜLLER S., 1998. Application de la méthode Milieu Et Végétaux aquatiques fixés à 12 rivières françaises: typologie floristique préliminaire. Annales de Limnologie, 34(2), 129-138. 
HENRY C.P., AMOROS C., 1996. Restoration ecology of riverine wetland. III. Vegetation survey and monitoring optimisation. Ecological Engineering, 7, 35-58.

JURKO A., 1986. Plant communities and some questions of their taxonomical diversity, Ekologia (CSSR), 5(1), 3-32.

KHALANSKI M., BONNET M., GRÉGOIRE A., 1990. Evaluation quantitative de la biomasse végétale en Durance à l'aval du barrage de Serre-Ponçon. Hydroécologie Appliquée, 1-2, 55-89.

KING J., CAMBRAY J.A., IMPSON N.D., 1998. Linked effects of dam-released floods and water temperature on spawning of the Clanwilliam yellowfish Barbus capensis. Hydrobiologia, 384, 245-265.

LEGENDRE L., LEGENDRE P., 1984. 2. La structure des données écologiques, Masson Presses de I'Université du Québec, Paris Québec, $335 \mathrm{p}$.

LEGLIZE L., PELTRE M.C., DECLOUX J.-P., DUVAL T., PARIS P., ZUMSTEIN J.-F., 1990. Caractérisation des milieux aquatiques d'eaux courantes et végétation fixée. 14ème conférence du COLUMA - journées internationales d'études sur la lutte contre les mauvaises herbes, Versailles, 237-245.

MADSEN J.D., ADAMS M.S., 1988. The seasonal biomass and productivity of the submerged macrophytes in a polluted Wisconsin stream. Freshwater Biology, 20, 41-50.

MAGURRAN A.E., 1988. Ecological diversity and its measurement, Chapman, London. $179 \mathrm{p}$.

MC CREARY N., 1991. Competition as a mechanism of submersed macrophyte community structure. Aquatic Botany, 41, 177-193.

MURTAUGH P.A., 1996. The statistical evaluation of ecological indicators. Ecological Applications, 6(1), 132-139.

NEWBOLD C., HOLMES N.T.H., 1987. Nature conservation : water quality criteria and plants as water quality monitors. Water Pollution Control, 86(2), 345-364.

PADMORE C.L., 1997. Biotopes and their hydraulics : a method for defining the physical component of freshwater quality. In : BOON P.J. and HOWELL D.L. (Eds), Freshwater quality : defining the indefinable? Her Majesty's Stationery Office, London, 251-257.

PETITJEAN F., 1981. Etude de l'herbier à Ranunculus fluitans d'Argentat sur la Dordogne, Thèse de l'Univ. Paul Sabatier, Toulouse, $188 \mathrm{p}$.

PIELOU E.C., 1975. Ecological diversity, John WILEY and SONS, New York. 165 p.

POTVIN C., ROFF D.A., 1993. Distribution-free and robust statistical methods : viable alternatives to parametric statistitics? Ecology, 74(6), 1617-1628.

PRYGIEL J., 1997. Biologie et écologie des espèces végétales proliférant en France. Les études de l'Agence de l'Eau n68, $199 \mathrm{p}$.

RAUNKIAER C., 1905. Types biologiques pour la géographie botanique. Bul. Acad. Roy. Sci. Denmark, 5, 347-437.

RECKHOW K.H., CLEMENTS J.T., DODD R.C., 1990. Statistical evaluation of mechanistic water-quality models. Journal of Environmental Engineering, 116(2), 250-268.

SHAW R.G., 1993. ANOVA for unbalanced data : an overview. Ecology, 74(6), 1638-1645.

STANDING COMMITTEE OF ANALYSTS, 1987. Methods for the use of aquatic macrophytes for assessing water quality 1985-86. Her Majesty's Stationery Office, London, $176 \mathrm{p}$.

WARD J.V., STANFORD J.A., 1983a. The serial discontinuity concept of lotic ecosystems. In : BARTELL F. (ed.), Dynamics of lotic ecosystems, Ann Arbor Science, 29-42. 
WARD J.V., STANFORD J.A., 1983b. The intermediate-disturbance hypothesis : an explanation for biotic diversity patterns in lotic ecosystems. In : BARTELL F. (ed.), Dynamics of lotic ecosystems, Ann Arbor Science, 347-356.

WILKINSON L., HILL M.A., 1994. SYSTAT for DOS : Using SYSTAT, version 7. SYSTAT, Inc Evanston, IL, $871 \mathrm{p}$. 